\title{
Simple spin-free calculations for small molecules
}

\author{
B L Burrows ${ }^{1}$, and M.Cohen ${ }^{2}$ \\ ${ }^{1}$ Mathematics Section \\ School of Creative Arts and Engineering \\ Staffordshire University, College Road, Stoke-on-Trent,ST4 2DE UK \\ e-mail:B.L.Burrows@staffs.ac.uk \\ ${ }^{2}$ Department of Physical Chemistry \\ The Hebrew University of Jerusalem \\ Jerusalem 91904, Israel \\ e-mail:maurice@fh.huji.ac.il
}

\begin{abstract}
A simple treatment of spin-free quantum theory is applied to 3-electron systems, constructing the matrix representations and the corresponding projection operators. The theory is used to treat two example molecules $\mathrm{HeH}$ using a model where an helium atom interacts first with a proton and then an electron; and linear $H_{3}$ where a basis of three 1s functions is used to calculate a set of allowed states. For both systems the energies compare well to earlier, more extensive, calculations.
\end{abstract}

\section{Introduction}

The usual description of physical systems (such as atoms and molecules) is based on a hybrid theory. Space variables appear explicitly in the conven- 
tional non-relativistic Schrödinger Hamiltonian, H, whereas there is no sign of spin coordinates. Just as it is necessary to impose some spatial boundary conditions, so is it essential to ensure that the Pauli principle (Pauli 1928) is satisfied so that spin effects, formally entirely absent from $\mathrm{H}$, are correctly accounted for. Historically, N-electron wave functions have almost always been described by a few determinants made up of single electron orbitals each presented as a product of a space function for a single electron (usually in ordinary three-dimensional space) and a spin-function, conventionally alpha or beta.Whilst this will suffice to satisfy the Pauli principle it does not explicitly impose the correct spatial and spin symmetries on the wavefunction. Here we use what has been termed spin-free quantum theory to impose the conditions. This is not a completely accurate name since although the energies are obtained entirely from the spatial Schrödinger equation the form of the wavefunction used is chosen so as to give the correct overall symmetry. The spin-free theory is reexamined in the next section and further details given in an appendix. Some earlier treatments of spin-free theory, for example Musher (1970) ,Amos (1973),Amos and Burrows (1970) and McWeeny (1988) are generally not well known; these include valence bond theory and calculations using both ab initio and perturbation theories. In our present study, we focus on some small 3-electron systems, in particular $\mathrm{HeH}$ and $\mathrm{H}_{3}$ which are of astrophysical interest. We do not seek spectroscopic accuracy, but rather good qualitative descriptions of the physics and chemistry of such systems using simple spin-free techniques. .

One of the most widely-used approximate wavefunctions is obtained using the approach of Heitler and London (1927) (HL) . Whilst there are many very good numerical solutions for simple systems that have been developed over the years HL remains a good qualitative picture of the interaction. In the traditional HL method for two-electron systems, simple variational calculations lead to wavefunctions symmetric or antisymmetric in interchange of the spatial coordinates and the Pauli principle is imposed by multiplying these by spin functions that are respectively antisymmetric or symmetric in the interchange of spin coordinates. When the theory is extended to 3 or more electrons the Pauli principle needs to be considered in more detail and this is illustrated in our calculations.In section 2 we examine some low-lying states of $\mathrm{HeH}$ and introduce a novel and simple model that gives results comparable to some earlier extensive variational calculations (Michels and Harris 1963). In the subsequent section we treat $H_{3}$ in a basis of three states each 
centred on a different atom, but allowing for the correct symmetry. As with the earlier example, this model gives energies comparable to more extensive calculations together with simple approximations to the corresponding wave functions.

\section{Symmetry Properties of the electronic Hamil- tonian}

We begin by considering the non-relativistic electronic Hamiltonian $\mathrm{H}$ ( for a fixed nuclear configuration) which is independent of spin and the corresponding Schrödinger equation takes the form

$$
H \psi=E \psi
$$

Since $\mathrm{H}$ is independent of spin, it is possible to solve equation (1) without reference to any spin properties but then we need to invoke the Pauli principle so that the physical wavefunction is antisymmetric in the interchange of any pair of electron coordinates. To form this wavefunction we need to augment the spatial wavefunction with spin functions in a way that ensures that we have such a physical wavefuntion.

For any permutation of the spatial electronic coordinates, $P^{r}$ say, since $\mathrm{H}$ is symmetric in all space coordinates, we have the commutation property

$$
\left[P^{r}, H\right]=0
$$

so that

$$
P^{r} H \psi=H\left(P^{r} \psi\right)
$$

$\mathrm{Eq}(3)$ implies that either the wavefunction $\psi$ is unchanged when the permutation operator $P^{r}$ is applied or equation 1 has degenerate solutions. Thus we may write the general solution for this particular $\mathrm{E}$ in the form

$$
\psi=\sum_{i=1}^{d} c_{i} \psi_{i}
$$

where the $\psi_{i}$ are $\mathrm{d}$ degenerate independent solutions. If a solution $\psi$ is unchanged by the action of all permutations then $d=1$. 
For any one of the degenerate solutions $\psi_{j}$ and any particular $P^{r}$ we have

$$
P^{r} \psi_{j}=\sum_{i=1}^{d} U_{i j}\left(P^{r}\right) \psi_{i}, \quad j=1 . . d
$$

where the $U_{i j}\left(P^{r}\right)$ are scalar coefficients. Thus for each permutation there will be a different set of $d^{2}$ coefficients but the required coefficients can be obtained entirely from a matrix representation of the permutation group of $\mathrm{N}$ objects ( $\mathrm{N}$ being the number of electrons).For example, for non-degenerate situations, $d=1$ and we have $U_{11}\left(P^{r}\right)=1$ for all the permutations. The allowed degeneracies resulting from the permutation symmetry ( there may be additional accidental degeneracies for a particular Hamiltonian) are determined from the matrix representation. These take into account that any set of degenerate functions can sometimes have the property that they can be divided into subsets so as the effect of acting on any given subset with all permutations gives rise only to members of that subset. Thus the degeneracy problem has been reduced to looking at the properties of the subsets. When the reduction has gone as far as possible we say that the subsets form a basis for an irreducible representation of the permutation group and any accidental degeneracy combines two or more of these irreducible representations. A procedure for constructing the matrices for these irreducible representation is described in Appendix 1. They can be classified using partitions of $\mathrm{N}$ : $\left[n_{1}, n_{2} \ldots n_{s}\right]$ where

$$
\sum_{i=1}^{s} n_{i}=N \quad i>j \Rightarrow n_{i} \leq n_{j}
$$

Consider any solution of the spin independent Schrödinger equation $\psi_{k}$ and operate on this function with the antisymmetrizer in $\mathrm{N}$ particles :

$$
A_{N}=\sum_{P} \delta_{p} P \psi_{k}
$$

where $\delta_{p}$ is the parity of the permutation $P$ and the sum is over all permutations. We obtain either a function that is antisymmetric in all the particles, or zero. In the former case $\psi_{k}$ has the symmetry properties of an irreducible representation of dimension 1 and there is no degeneracy. If we obtain zero, we can operate with an antisymmetrizer in $\mathrm{N}-1$ particles and if this is non zero we can clearly obtain N-1 degenerate states ( one for each electron coordinate omitted from the antisymmetrizer). If we have zero we can continue the process so that finally we obtain a state of maximal antisymmetry in 
$n_{1}$ particles, maximal antisymmetry in the next $n_{2}$ particles and so on producing the partition $\left[n_{1}, n_{2} \ldots n_{s}\right]$. Thus the degeneracies are classified by the corresponding partition. These are often illustrated using Young Tableau where the columns of non-increasing length illustrate the analysis by antisymmetry. Of course the length of the rows also define a partition and this corresponds to the fact the symmetry can be used to classify the irreducible representations instead of antisymmetry.

\subsection{The symmetry of the spin functions}

Since $\mathrm{H}$ is spin-independent any permutation $P^{\sigma}$ of the spin variables commutes with $\mathrm{H}$ and the set $\left\{P^{\sigma}\right\}$ also may be classified using the irreducible representations of $\mathrm{N}$ particles, but in order to satisfy the Pauli principle there is a relationship between the representations of the space coordinates and the spin coordinates. However, the N-particle spin-functions are constructed from strings of one particle functions $\alpha$ or $\beta$ and cannot be antisymmetrized in more than two coordinates. This restricts the representations that can produce physically allowed solutions. To amplify this, for any set of independent degenerate space functions $\left\{\psi_{i}\right\}$ associated with an irreducible representation we can augment them by spin functions $\theta_{i}$ to be determined, so that the total wavefunction is

$$
\Psi=\sum_{i=1}^{d} \psi_{i} \theta_{i}
$$

The Pauli principle may be expressed as

$$
P \Psi=\delta_{p} \Psi, \quad P=P^{r} P^{\sigma} \quad\left(\delta_{p}= \pm 1\right)
$$

where now $P^{\sigma}$ is the identical permutation of the spin function as $P^{r}$ for the space function. Thus we require

$$
\Psi=\delta_{p} \sum_{i=1}^{d} P^{r} \psi_{i} P^{\sigma} \theta_{i}=\delta_{p} \sum_{i, j}^{d} U_{j i}^{r}\left(P^{r}\right) \psi_{j} P^{\sigma} \theta_{i}
$$

using (6). The $\psi_{j}$ are independent so we may deduce that

$$
\theta_{j}=\delta_{p} \sum_{i}^{d} U_{j i}^{r}\left(P^{\sigma}\right) P^{\sigma} \theta_{i}
$$


where $P^{\sigma}$ is the same permutation as $P^{r}$ and consequently the matrix elements of $U$ are the same.This determines the corresponding spin functions and we may deduce that

$$
\left(P^{\sigma}\right)^{-1} \theta_{j}=\delta_{p} \sum_{i}^{d} U_{j i}^{r}\left(P^{\sigma}\right) \theta_{i}
$$

Since $\left(P^{\sigma}\right)^{-1}$ is an arbitrary permutation this shows that the corresponding spin functions belong to another representation, called the dual representation, where the matrix elements for any permutation are related by

$$
U_{i j}^{\sigma}\left(P^{\sigma}\right)=\delta_{p} U^{r}\left(\left(P^{\sigma}\right)^{-1}\right)_{j i}
$$

for any $P^{\sigma}$. The properties of the matrices depend on the basis used for the matrix representation but ,for convenience, we choose a real and orthonormal basis which implies that $\mathrm{U}$ is an orthogonal matrix and

$$
U^{T}(P)=U^{-1}(P)=U\left(P^{-1}\right)
$$

Thus the relationship between the representation matrices for the spatial functions and dual spin-functions is

$$
\left(U^{\sigma}\left(P^{\sigma}\right)\right)^{T}=\delta_{p}\left(U^{r}\left(P^{r}\right)^{-1}\right)
$$

using the relationship between the matrices for the representations and their dual. Since $\mathrm{P}$ and the inverse $P^{-1}$ are arbitrary permutations this shows that choosing the spin functions from the dual representation ensures that the Pauli principle is satisfied but the eigenvalue can be found from the Schrödinger equation from any one of the degenerate space functions.

\section{An Application to $\mathrm{HeH}$}

Here we consider the three-electron system $\mathrm{HeH}$. There are two possible physically allowed spatial irreducible representations. One is the completely antisymmetric spacial wavefunction ( corresponding to a symmetric spin function). The other is the two-dimensional representation examined in Appendix 1 where the spatial wavefunctions satisfy

$$
\frac{1}{3}\left(1-\frac{1}{2}\left(P_{13}^{r}+P_{23}^{r}\right)\right)\left(1+P_{12}^{r}\right) \Psi_{1}(1,2,3)=\Psi_{1}(1,2,3)
$$


and

$$
\frac{1}{3}\left(1+\frac{1}{2}\left(P_{13}^{r}+P_{23}^{r}\right)\right)\left(1-P_{12}^{r}\right) \Psi_{2}(1,2,3)=\Psi_{2}(1,2,3)
$$

We note that, in principle,we can use either of these two forms to find the eigenvalue $\mathrm{E}$ but if we make approximations in the calculation then these may give different estimates. In order to construct a generalised HL approximation for $\mathrm{HeH}$ we will consider the symmetry at infinite separation and use the product of a 1 s function centred on the hydrogen atom and a function symmetric in the interchange of two electrons centred on the Helium atom. Such a product function will be a good approximation for large internuclear separations where the interchange effects can be ignored, but can be adjusted to satisfy the Pauli principle at finite $\mathrm{R}$ by using the projection operator $T_{1}$ above. Thus the approximation may be written in the form

$$
\Psi=T_{1} \psi(1,2, R) \phi(3)=\frac{1}{3}\left(1-\frac{1}{2}\left(P_{13}^{r}+P_{23}^{r}\right)\right)\left(1+P_{12}^{r}\right) \psi(1,2, R) \phi(3)
$$

where

$$
\psi(1,2, R)=\psi(2,1, R)
$$

We will also specify that

$$
\lim _{R \rightarrow \infty} \psi(1,2, R)
$$

is an approximation to the ground state of He. This could be any such approximation in principle but for a generalisation of the HL procedure we take a product of one electron functions, centred at the He atom. Here we use such an atomic product function that also interacts with the proton $\mathrm{H}^{+}$ and so is an approximate wavefunction for $\mathrm{HeH}^{+}$. Once $\psi(1,2, \mathrm{R})$ has been obtained for a specified $\mathrm{R}$ we use a generalisation of the HL procedure by varying $\phi(3)$ using a simple one-parameter variational method to obtain an approximate $\mathrm{E}$ in the form

$$
E \approx \frac{<\Psi \mid H \Psi>}{<\Psi \mid \Psi>}
$$

where we specify that the helium atom is at $\mathrm{A}$ and the hydrogen atom is at $\mathrm{B}$ and

$$
H=h-\frac{1}{2} \nabla_{3}^{2}+\frac{1}{r_{13}}+\frac{1}{r_{23}}-\frac{2}{r_{3 a}}-\frac{1}{r_{3 b}}
$$


where

$$
h=-\frac{1}{2}\left(\nabla_{1}^{2}+\nabla_{2}^{2}\right)+\frac{2}{R}+\frac{1}{r_{12}}-\frac{2}{r_{1 a}}-\frac{1}{r_{1 b}}-\frac{2}{r_{2 a}}-\frac{1}{r_{2 b}}
$$

Note that $\mathrm{h}$ is a two-electron hamiltonian. The calculation can be simplified after $\psi(1,2, R)$ is obtained and we will now examine this.

\section{The approximate wavefunction for $\mathrm{HeH}^{+}$}

We form an approximation

$$
\psi(1,2, R)=\frac{1}{2}\left(1+P_{12}^{r}\right) \exp \left(-2.15 r_{1}\right) \exp \left(-R\left(\gamma p_{2}+\delta q_{2}\right) / 2\right)
$$

where $\gamma, \delta$ are variational parameters, depending on $\mathrm{R}$, chosen to minimise

$$
\frac{<\psi(1,2, R) \mid h \psi(1,2, R)>}{<\psi(1,2, R) \mid \psi(1,2, R)>}
$$

and where the one-electron exponential functions are centred on the Helium atom at $\mathrm{A}$. Here $p_{2}$, and $q_{2}$ are spheroidal coordinates defined by

$$
p_{2}=\frac{r_{2 a}+r_{2 b}}{R}, \quad q_{2}=\frac{r_{2 a}-r_{2 b}}{R}
$$

The results are given in table 1. Some earlier results for this ion (Michels and Harris 1963) are compared with our estimates and there is agreement to less than one percent at all separations $\mathrm{R}$ similar to the accuracy for atomic He (as $R \rightarrow \infty$ ). This is another generalisation of HL where now both electrons are centred on He. Since the helium atom has strongly bound electrons the interaction with the proton is small for a large range of $R$ the energy obtained is in line with typical calculations for the helium atom with a simple approximation which approaches

$$
\psi(1,2, \infty)=\frac{1}{2}\left(1+P_{12}^{r}\right) \exp \left(-2.15 r_{1}\right) \exp \left(-1.19 r_{2}\right)
$$

as the atoms separate ( Pauling and Bright Wilson 1935). The energy for the helium atom using this function is correct to a few percent and it is well known that to get an improvement we would need to include terms in $r_{12}$ but that would necessarily complicate the simple description. Traditional HL calculations are better for large $\mathrm{R}$ but the function chosen here, with the interaction with the proton included is designed to correct for small R.We note that for $R=0.001, E-2 / R=-7.247$ and a good estimate for the energy of $\mathrm{Li}^{+}$is -7.28 so that the wavefunctions obtained give a good qualitative result for a wide range of $R$. 
Table 1: The Spheroidal Variational Energies and Parameters $\mathrm{HeH}+$

\begin{tabular}{|c|c|c|c|c|}
$\mathrm{R}$ & Michels and Harris & This calculation & $\gamma$ & $\delta$ \\
20 & - & -2.875 & 1.20 & 1.18 \\
10 & - & -2.875 & 1.21 & 1.18 \\
8 & - & -2.875 & 1.23 & 1.18 \\
6 & -2.876 & -2.876 & 1.23 & 1.15 \\
4 & -2.879 & -2.879 & 1.27 & 1.08 \\
3 & -2.888 & -2.886 & 1.32 & 0.940 \\
2 & -2.921 & -2.917 & 1.46 & 0.55 \\
1.75 & -2.934 & -2.931 & 1.52 & 0.40 \\
1.4 & -2.943 & -2.941 & 1.65 & 0.20 \\
1.25 & -2.934 & -2.932 & 1.68 & 0.11 \\
1 & -2.871 & -2.870 & 1.87 & -0.02 \\
0.5 & - & -1.901 & 2.39 & 0.01 \\
0.1 & - & 12.908 & 2.94 & 0.05 \\
0.001 & - & 1992.77 & 3.00 & 0.02
\end{tabular}

\subsection{A Spin-free model for the states of $\mathrm{HeH}$}

The generalised HL energies are obtained by evaluating eq (21) for specific values of $\mathrm{R}$, making use of the parameters in Table 1 . Using the operator $T_{1}$ defined in (18) we may specify that $\Psi$ belongs to this particular irreducible representation rewrite $(21)$ in the form

$$
E \approx \frac{<T_{1} \Psi \mid H \Psi>}{<T_{1} \Psi \mid \Psi>}
$$

If we have the exact wavefunction for $\mathrm{HeH}^{+}$with energy $\mathrm{E}_{0}$ then (28) may be explicitly expressed in the form

$$
E\left(<T_{1} \Psi \mid \Psi>\right) \approx<T_{1} \Psi \mid\left(E_{0}-\frac{1}{2} \nabla_{3}^{2}+\frac{1}{r_{13}}+\frac{1}{r_{23}}-\frac{2}{r_{3 a}}-\frac{1}{r_{3 b}}\right) \Psi>
$$

To evaluate $\mathrm{E}$ we use an approximation for the exact functions as defined in (23) and the values are listed in table 1 . This corresponds to a model with a fixed $\mathrm{HeH}^{+}$core and varying $\phi$ which depends on a single variational parameter t. The model is justified since $\mathrm{HeH}$ is known to be a Rydberg 
Table 2: Energies and Parameters for the ground state of $\mathrm{HeH}$

molecule where the outer electron behaves like an hydrogen-like orbital relative to the ionic core. Thus, in the case of the ground state, an appropriate form for the trial function $\phi$ is

$$
\phi=\exp (-t r)=\exp (-t R(p-q) / 2)
$$

where $\mathrm{p}$ and $\mathrm{q}$ are the spheroidal coordinates and the one-electron trial function is centred at the $\mathrm{H}$ atom. The results are presented in Table 2 and there is agreement to about 1 percent with the earlier, more sophisticated, calculations by Michels and Harris (1963). We note that the accuracy must always be limited by the accuracy of the fixed ionic core. For large $\mathrm{R}$ the polarization effects from the proton will be small and in these cases the calculated energies using standard approximation for atomic helium is approximately 1 percent ,so that our results cannot be more accurate unless we extend the helium core approximation. To improve the accuracy we would need to include terms in $r_{12}$ or in the azimuthal angles.For smaller $\mathrm{R}$ the results may be less accurate, but with $R=0.001$ we obtain

$$
E-\frac{1}{R}=-7.4
$$

which compares well with the approximate united $\mathrm{Li}$ atomic value of -7.478 . The model used cannot be expected to treat atomic Lithium accurately; spherical coordinates centred on the He atom would be more efficient.

In order to treat excited states we have used two different choices of $\phi$. These were

$$
\phi_{1}=\exp \left(-t_{1} R(p-q) / 2\right)(1+A p)
$$


Table 3: Excited states with a node in $\mathrm{p}$

or

\begin{tabular}{|c|c|c|c|}
$\mathrm{R}$ & Michels and Harris & This calculation & $t_{1}$ \\
10 & - & -2.999 & 0.51 \\
6 & -3.001 & -2.999 & 0.51 \\
4 & -3.003 & -3.000 & 0.51 \\
3 & -3.011 & -3.007 & 0.50 \\
2 & -3.044 & -3.035 & 0.49 \\
1.4 & - & -3.007 & 0.27 \\
1.25 & -3.064 & -2.996 & 0.25 \\
1 & -3.002 & -2.930. & 0.23
\end{tabular}

$$
\phi_{2}=\exp \left(-t_{2} R(p-q) / 2\right)(1+B q)
$$

The values of $A, B$ are chosen so that these wavefunctions are orthogonal to the approximate ground state wavefunction of $\phi$ for the same value of $R$. In this way we obtain three mutually independent functions, each of which is a one parameter function. The corresponding energies are given in tables 3 and 4 and are also compared with some earlier values calculated by Michels and Harris (1963) .We note that for large R, these excited state wavefunctions approach degenerate excited states of hydrogen expressed in parabolic coordinates and are not pure $\mathrm{S}$ or $\mathrm{P}$ states.

The energy values for E are subject to any numerical errors in the calculation of the two-electron integrals. The method of estimating these is based on the Neumann expansion of $1 / r_{12}$ in terms of Legendre functions in spheroidal coordinates .( See Rüdenberg 1951 for a derivation).

Although our results are slightly less accurate than extensive numerical calculations, they nevertheless provide a good physical description for all R. For large $\mathrm{R}$ more terms are needed in the expansion and the accuracy depends on the variational approximation for $\mathrm{HeH}^{+}$. 
Table 4: Excited states with a node in $\mathrm{q}$

\begin{tabular}{|c|c|c|c|}
$\mathrm{R}$ & Michels and Harris & This calculation & $t_{2}$ \\
10 & -3.000 & -2.992 & 0.48 \\
6 & - & -2.964 & 0.48 \\
4 & -3.003 & -2.953 & 0.18 \\
3 & -3.010 & -2.968 & 0.19 \\
2 & -3.048 & -3.027 & 0.49 \\
1.4 & - & -3.093 & 0.38 \\
1.25 & -3.081 & -3.084 & 0.38 \\
1 & -3.022 & -3.016. & 0.36
\end{tabular}

\section{Allowed and Un-allowed Physical States}

In a calculation using a basis of spatial functions the effect of the Pauli principle is to reduce severely the number of possible configurations which need to be included. To illustrate this we consider a simple model for linear $\mathrm{H}_{3}$ where the basis consists of three functions each centred on a different atom. Denoting these by a,b and c centred on atoms A, B and C respectively the basis is the set $\left\{\phi_{i}(1) \phi_{j}(2) \phi_{k}(3)\right\}$ where each $\phi_{i}$ is one of a, b or c and consequently there are a total of 27 basis functions. The three functions are chosen by considering the isolated atoms in the limit as the outer atoms move away from the centre so that we use 1s hydrogen-like ground state orbitals centred on atoms $\mathrm{A}, \mathrm{B}$ and $\mathrm{C}$ respectively. The Hamiltonian for this system is similar to that for $\mathrm{HeH}$ given in (22), except for the term in $1 / R$, but the wave function has a different form as $R \rightarrow \infty$ so that

$$
\Psi=\sum_{i j k} a_{i j k} \phi_{i} \phi_{j} \phi_{k}
$$

The corresponding matrix elements are $<r s t|H| m n l>$ and the eigenvalues are given in table 5 for the case $R=3.778$ so as to compare the ground state energy obtained in an earlier calculation of Hirschfelder et al (1935). The wavefunctions belong to one of the irreducible representations and taking into account the Pauli Principle, only the representations corresponding to the partitions $[2,1]$ or $[3]$ give rise to allowed physical states. In table 5 the states are classified into allowed and un-allowed states. The completely 
antisymmetric state corresponding to the partition [3] is marked as (A). The other allowed states in the first column are each degenerate corresponding to the partition $[2,1]$. The un-allowed states correspond to the partition $[1,1,1]$. This classification can be carried out using antisymmetrizing operators, but there is a simpler technique which also reduces the amount of work necessary to calculate the allowed physical states.. For each degenerate state we may choose the symmetry so that the solution is antisymmetric in the interchange of variables 1 and 2 and together with the completely antisymmetric solution we consider only the matrix in a basis for this set. To construct such a basis we calculate

$$
\chi_{i j k}=\left(1-P_{12}\right) \phi_{i} \phi_{j} \phi_{k}
$$

and the resulting 9 non-zero functions $\chi_{i j k}$ form the required basis. The eigenvalues of the corresponding matrix enable all the distinct, physically allowed energies to be calculated which , taking into account the degeneracy,give rise to 17 physically allowed states.Since the functions in this adapted basis are in the space of the allowed solutions, the ground state energy is a lower bound for these energies.

The calculated lowest allowed state in table 5 is -1.596 when $R=3.778$ which compares with -1.589, in an earlier, pioneering calculation of Hirschfelder et al (1935) .Some later large multi-cconfiguration calculations at $R=3.6$ (Considine and Heyes 1966 , Edmiston and Krauss 1964) give the results -1.612 and -1.648 respectively so that our calculations have comparable accuracy in energy together with a simple representation of the wavefunction. Here, in addition, we have obtained a set of excited states which illustrates the structure of the spectrum in this basis. 
Table 5: Allowed and Un-allowed States for $\mathrm{H}_{3}$

\begin{tabular}{|l|c|} 
Allowed States & Un-allowed States \\
- & 0.531 \\
-0.064 & 0.421 \\
-0.307 & 0.195 \\
-0.521 & -0.263 \\
-0.704 & -0.330 \\
-0.727 & -0.743 \\
-1.038 & -0.876 \\
$-1.059(\mathrm{~A})$ & -1.065 \\
-1.341 & -1.298 \\
-1.596 & -1.736
\end{tabular}

\section{Appendix 1: The construction of the represen- tation}

The irreducible matrix representations are not unique and a particular, nonunique procedure has been given by Hamermesh (1962). Here we illustrate a procedure for constructing the matrices for $N=3$. (For the special case of $N=2$ there is no degeneracy and there are two irreducible representations each of dimension 1 leading to the wavefunctions being symmetric or antisymmetric in the space coordinates). We first find the representations for the spin permutations from which,using (15), we can immediately find the corresponding matrices for the space permutations. To do this we follow a technique introduced by Amos (1973), making use of the spin annihilation operator $S_{-1}$ which is given by

$$
S_{-1}=\sum_{i=1}^{N} s_{-1}(i), \quad s_{-1}(i) \alpha(i)=\beta(i), s_{-1}(i) \beta(i)=0
$$

Here $\alpha(i), \beta(i)$ are the usual spin functions and the same analysis can be carried out using $S_{+}$. Since $S_{-1}$ is symmetric in the spin coordinates it commutes with all the spin permutation operators so that given the representation where

$$
P^{\sigma} \Theta_{i}=\sum_{j} U_{j i} \Theta_{j}
$$


where the dependence of $\mathrm{P}$ in the matrix elements has been omitted and with $\left\{\theta_{i}\right\}$ being the representative functions then

$$
S_{-1} P^{\sigma}=P^{\sigma} S_{-1} \Rightarrow P^{\sigma}\left(S_{-1} \Theta_{i}\right)=\sum_{P} U_{j i}\left(S_{-1} \Theta_{j}\right)
$$

That is $S_{-1} \Theta_{i}$ and $\Theta_{i}$ have exactly the same transformation properties.

Applying $S_{-1}$ to the spin function $\alpha(1) \alpha(2) \alpha(3)$ and continuing the application gives the following functions up to multiplicative constants

$$
\alpha \alpha \alpha \rightarrow \alpha \alpha \beta+\alpha \beta \alpha+\beta \alpha \alpha \rightarrow \alpha \beta \beta+\beta \alpha \beta+\beta \beta \alpha \rightarrow \beta \beta \beta
$$

where the coordinate labels 1,2,3 have been omitted. All these have the same transformation properties and since the first one is one dimensional all are 1 dimensional irreducible representations and the action of any permutation on these functions just give a multiple of them. The second and third of these cases give rise to other representations since for example in the second case we may construct two spin-functions orthogonal to $\alpha \alpha \beta+\alpha \beta \alpha+\beta \alpha \alpha$ and since the action of any permutation on them produces a combination of these two functions; that is they form a basis for a two-dimensional irreducible representation. There are clearly many ways to do this but it is convenient to choose the normalized functions

$$
\phi_{1}=\frac{1}{\sqrt{2}}(\alpha \beta-\beta \alpha) \alpha, \quad \phi_{2}=\frac{1}{\sqrt{6}}(2 \alpha \alpha \beta-(\alpha \beta+\beta \alpha) \alpha)
$$

Note that these two orthonormal functions are respectively antisymmetric and symmetric in the interchange of variables 1 and 2 . We now calculate the two-dimensional matrix representation by calculating $<\phi_{i} P \phi_{j}>, i, j=1,2$ where $P=P_{12}^{\sigma}, P_{23}^{\sigma}$. All the other matrices can be defined by matrix multiplication since in general $P_{k k+1}^{\sigma}, \quad k=1,2$.. generate all the permutations.( For example $\left.P_{13}^{\sigma}=P_{12}^{\sigma} P_{23}^{\sigma} P_{12}^{\sigma}\right)$. The matrices for $P_{12}^{\sigma}, P_{23}^{\sigma}, P_{13}^{\sigma}$ are:

$$
\begin{gathered}
U^{\sigma}\left(P_{12}^{\sigma}\right)=\left(\begin{array}{cc}
-1 & 0 \\
0 & 1
\end{array}\right), U^{\sigma}\left(P_{23}^{\sigma}\right)=\left(\begin{array}{cc}
1 / 2 & \sqrt{3} / 2 \\
\sqrt{3} / 2 & -1 / 2
\end{array}\right) \\
U^{\sigma}\left(P_{13}^{\sigma}\right)=\left(\begin{array}{cc}
1 / 2 & -\sqrt{3} / 2 \\
-\sqrt{3} / 2 & -1 / 2
\end{array}\right)
\end{gathered}
$$


Using the relationship between the matrices for the spin and corresponding space irreducible representations , (15), we can write down the corresponding space matrices. Here our representations are real.

$$
\begin{gathered}
U^{r}\left(P_{12}^{r}\right)=\left(\begin{array}{cc}
1 & 0 \\
0 & -1
\end{array}\right), U^{r}\left(P_{23}^{r}\right)=\left(\begin{array}{cc}
-1 / 2 & -\sqrt{3} / 2 \\
-\sqrt{3} / 2 & 1 / 2
\end{array}\right) \\
U^{r}\left(P_{13}^{r}\right)=\left(\begin{array}{cc}
-1 / 2 & \sqrt{3} / 2 \\
\sqrt{3} / 2 & 1 / 2
\end{array}\right)
\end{gathered}
$$

From these we may deduce the following symmetry properties of the corresponding space functions , $\psi_{1}, \psi_{2}$,

$$
\begin{aligned}
& P_{12}^{r} \psi_{1}=\psi_{1}, \quad\left(P_{23}^{r}+P_{13}^{r}\right) \psi_{1}=-\psi_{1} \\
& P_{12}^{r} \psi_{2}=-\psi_{2}, \quad\left(P_{23}^{r}+P_{13}^{r}\right) \psi_{2}=\psi_{2}
\end{aligned}
$$

We now construct projection operators specific to this irreducible representation. . For example choosing

$$
T_{11}=\left(1-P_{13}^{r}\right)\left(1+P_{12}^{r}\right), T_{12}=\left(1-P_{23}^{r}\right)\left(1+P_{12}^{r}\right)
$$

we obtain two operators that project onto this irreducible representation with partition $[2,1]$ so that the wavefunction can be antisymmetrical in at most 2 electrons.

Adding the same multiple of $T_{11}$ and $T_{12}$ gives

$$
T_{1}=k\left(2-P_{13}^{r}-P_{23}^{r}\right)\left(1+P_{12}^{r}\right)
$$

and choosing $k=1 / 6$ leads to

$$
T_{1}=\frac{1}{3}\left(1-\frac{1}{2}\left(P_{13}^{r}+P_{23}^{r}\right)\right)\left(1+P_{12}^{r}\right) . \quad T_{1} \psi_{1}=\psi_{1}, \quad T_{1} \psi_{2}=0
$$

The corresponding projection operator for $\psi_{2}$ is

$$
T_{2}=\frac{1}{3}\left(\left(1-P_{12}^{r}\right)+\frac{1}{2}\left(P_{23}^{r}+P_{13}^{r}\right)\left(1-P_{12}^{r}\right)\right)
$$


Note that $T_{1}$ can be written in the forms

$$
T_{1}=\frac{1}{6}(A(1,3)+A(2,3)) S(1,2)=\frac{1}{6} S(1,2)(A(1,3)+A(2,3))
$$

where $A(a, b)$ is the anti-symmetrizer of electrons with coordinates $a, b$ and similarly $S(a, b)$ is the symmetrizer defined by

$$
A(a, b)=\frac{1}{2}\left(1-P_{a b}\right), \quad S(a, b)=\frac{1}{2}\left(1+P_{a b}\right)
$$

Functions transforming according to the other two representations in this case satisfy

$$
A(1,2,3) \phi_{1}=\phi_{1}
$$

and

$$
S(1,2,3) \phi_{2}=\phi_{2}
$$

where

$$
\begin{aligned}
& A(a, b, c)=\frac{1}{6}\left(1-P_{a b}-P_{a c}-P_{b c}+P_{a b c}+P_{b a c}\right), \\
& S(a, b, c)=\frac{1}{6}\left(1+P_{a b}+P_{a c}+P_{b c}+P_{a b c}+P_{b a c}\right)
\end{aligned}
$$

Furthermore using the property that the weights of $P$ and $P^{-1}$ are the same in every antisymmetrizer $\mathrm{A}$ and symmetrizer $\mathrm{S}$ then since

$$
<f\left|P g>=<P^{-1} f\right| g>
$$

for any functions $f, g$ we may deduce that

$$
<f|A g>=<A f| g>, \quad<f|S g>=<S f| g>
$$

With these results we see that operating by $T_{1}$ on a general function $\Psi$ annihilates all components other than $\psi_{1}$ and consequently

$$
T_{1} \Psi=\mu \psi_{1}
$$

for some constant $\mu$. 


\section{References}

Amos A.T. (1973),Wave Mechanics: The first fifty years,J.Wiley, New York,119

Amos A.T. and Burrows B.L. (1970), J.Chem.Phys.53 ,939 1970

Considine J .P and Hayes E.F.(1966), J.Chem. Phys. 46,1119

Edmiston C. and Krauss M.J,(1964), J.Chem.Phys. 42, 121

Hamermesh M.(1962), Group Theory and its applications to physical problems,Addison-

Wesley London

Heitler W. and London F. (1927), Zeits.f.Physik, 44 ,455

Hirschfelder, J. ,Eyring H. and Rosen N. (1935), J.Chem. Phys. 4, 121

McWeeny R. (1988), Int. J.Quant.Chem., 34,25

Michels H.H and Harris F.E.(1963), J.Chem. Phys. 391464

Musher J.I. ( 1970), J. Phys( Paris) , 31 ,C4

Pauli W. (1925), Z.Phys. 31765

Pauling L. and Bright Wilson E. (1935), Introduction to Quantum Mechanics,,McGraw -Hill London, 223

Rüdenberg K. (1951),J.Chem.Phys. 19, 1459 